\title{
The Locus Ceruleus Is Involved in the Successful Retrieval of Emotional Memories in Humans
}

\author{
Virginie Sterpenich, ${ }^{1}$ Arnaud D'Argembeau, ${ }^{2}$ Martin Desseilles, ${ }^{1,4}$ Evelyne Balteau, ${ }^{1}$ Geneviève Albouy, ${ }^{1}$ \\ Gilles Vandewalle, ${ }^{1}$ Christian Degueldre, ${ }^{1}$ André Luxen, ${ }^{1}$ Fabienne Collette, ${ }^{1,2}$ and Pierre Maquet ${ }^{1,3}$ \\ ${ }^{1}$ Cyclotron Research Centre and ${ }^{2}$ Department of Cognitive Sciences, University of Liège, and ${ }^{3}$ Departments of Neurology and ${ }^{4}$ Psychiatry, Centre Hospitalier \\ Universitaire du Sart Tilman, B-4000 Liège, Belgium
}

\begin{abstract}
Emotional memories are better remembered than neutral ones. The amygdala is involved in this enhancement not only by modulating the hippocampal activity, but possibly also by modulating central arousal. Using functional magnetic resonance imaging, we analyzed the retrieval of neutral faces encoded in emotional or neutral contexts. The pupillary size measured during encoding was used as a modulator of brain responses during retrieval. The interaction between emotion and memory showed significant responses in a set of areas, including the amygdala and parahippocampal gyrus. These areas responded significantly more for correctly remembered faces encoded in an emotional, compared with neutral, context. The same interaction conducted on responses modulated by the pupillary size revealed an area of the dorsal tegmentum of the ponto-mesencephalic region, consistent with the locus ceruleus. Moreover, a psychophysiological interaction showed that amygdalar responses were more tightly related to those of the locus ceruleus when remembering faces that had been encoded in an emotional, rather than neutral, context. These findings suggest that the restoration of a central arousal similar to encoding takes part in the successful retrieval of neutral events learned in an emotional context.
\end{abstract}

Key words: emotion; memory; locus ceruleus; amygdala; pupillary size; functional magnetic resonance imaging

\section{Introduction}

Emotional events are usually better remembered than neutral ones (Dolan, 2002). The amygdala is involved in this enhancement of memory for emotional events. During encoding, the amygdala response to emotional stimuli (Cahill et al., 1996; Hamann et al., 1999) or neutral stimuli encoded in emotional context (Erk et al., 2003) is related to subsequent recall at a later date. Similarly, retrieving emotional memories is accompanied by significant amygdalar responses (Dolan et al., 2000), even when stimuli are intrinsically neutral but have been encoded in an emotional context (Maratos et al., 2001; Smith et al., 2004). In contrast, emotional memories are blunted by amygdalar lesions $(\mathrm{Ca}-$ hill et al., 1995; Adolphs et al., 1997).

The bias toward a better recollection of emotional events has been primarily explained by a direct modulation of hippocampal responses by the amygdala, at encoding (Kilpatrick and Cahill, 2003; Dolcos et al., 2004; Richardson et al., 2004) and during retrieval (Strange and Dolan, 2004; Fenker et al., 2005).

However, the amygdala might also improve the retrieval of emotional memories indirectly, by modulating central arousal (Davis and Whalen, 2001), defined as an activation of the forebrain by the activating structures of the brainstem and dienceph-

\footnotetext{
Received March 7, 2006; revised May 9, 2006; accepted June 4, 2006.

This work was supported by the Belgian Fonds National de la Recherche Scientifique (FNRS), La Fondation Médicale Reine Elisabeth, the University of Liège, and Pole d'attraction interuniversitaire P5/04. V.S., A.D'A., M.D., E.B., G.V., F.C., and P.M. were supported by the FNRS.

Correspondence should be addressed to Pierre Maquet, Centre de Recherches du Cyclotron, Université de Liège, B30, Sart Tilman, B-4000 Liège, Belgium. E-mail: pmaquet@ulg.ac.be.

DOI:10.1523/JNEUROSCI.1001-06.2006

Copyright $\odot 2006$ Society for Neuroscience $\quad$ 0270-6474/06/267416-08\$15.00/0
}

alon. The amygdala is in position to modulate central arousal through its projections to some of these target structures (Berntson et al., 2003; Pitkanen, 2004).

Increase in central arousal is usually accompanied by autonomic responses, which the amygdala is also in position to influence, through its direct projections to the hypothalamus (LeDoux et al., 1988) and brainstem (Davis, 1992). These autonomic responses can be used to assess the emotionally related arousing response in cognitive tasks. Typically, functional neuroimaging studies that estimated autonomic arousal relied on the measurement of galvanic skin conductance (Phelps et al., 2001; Williams et al., 2001) or heart rate (Critchley et al., 2005a). Changes in pupillary diameter are much faster than these two other measures, and allow the faithful assessment of emotional arousal specifically for each item presented. A study using the pupillary diameter found a correlation between the responses in the anterior cingulate cortex and the generation of autonomic arousal during a Stroop inhibitory task (Critchley et al., 2005b). To the best of our knowledge, the pupillary diameter was never used as a measure of emotional arousal in the framework of emotional memory.

In this study, we tested the hypothesis that successful recognition of stimuli encoded in emotional context is accompanied by a significant response not only in the amygdala but also in central arousing structures of the brainstem and the diencephalon. In addition, we reasoned that central arousing responses at retrieval, as a constituent element of the emotional memory, should be related to the arousal induced during encoding. 


\section{Encoding (40x)}

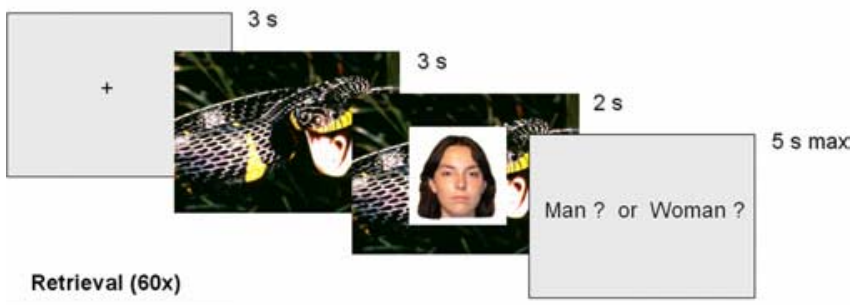

$3 \mathrm{~s}$

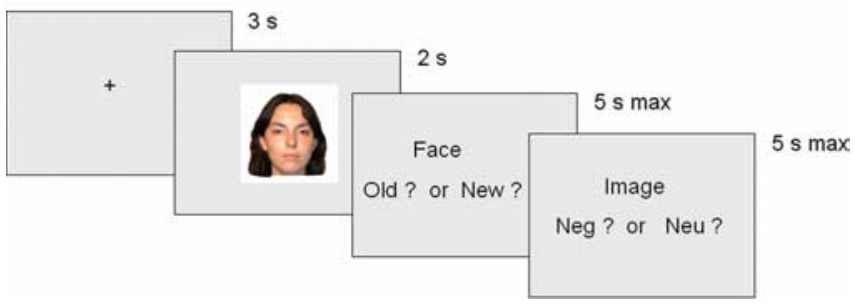

Figure 1. Experimental design.

\section{Materials and Methods}

Subjects. Thirty right-handed normal-sighted healthy volunteers ( $16 \mathrm{fe}-$ males; mean age, $22.3 \pm 2.6$ years) gave their written informed consent to take part in this functional magnetic resonance imaging (fMRI) study, which was approved by the Ethics Committee of the Faculty of Medicine of the University of Liège. None had medical, psychiatric, or traumatic history. Their scores on the Beck depression scale (Beck et al., 1961) and on the Bermond-Vorst Alexithymia Questionnaire (Bermond et al., 1999) were within normal ranges.

Behavioral task. Each subject was scanned during two separate sessions (Fig. 1). During the first session (encoding), each trial consisted of a background image $\left(17^{\circ} \times 23^{\circ}\right)$ displayed for $3 \mathrm{~s}$, on which a neutral face $\left(5^{\circ} \times 5^{\circ}\right)$ was superimposed centrally for $2 \mathrm{~s}$. After the stimuli disappeared, the subjects had a maximum of $5 \mathrm{~s}$ to categorize the face on the basis of its gender by pressing one of two possible keys on a keypad held in their right hand.

The retrieval session followed encoding after a delay of $5 \mathrm{~min}$, during which a distracting task (serial subtraction task) was performed. During the retrieval session, only neutral faces were displayed centrally for $1.5 \mathrm{~s}$, with 40 previously seen ("old") faces randomly mixed with 20 new neutral ("new") faces. The task consisted in a forced choice (yes/no) recognition task. The subject had $5 \mathrm{~s}$ to respond by a press on the keypad. If the face was recognized, the subject was further asked to specify whether it had been encoded in a neutral or emotional context (as set by the background image).

The analysis of behavioral data were performed with paired $t$ test on the percentage of remembered faces encoded in emotional or neutral contexts.

Experimental material. The set of background images was taken from the International Affective Pictorial System (Lang et al., 1999). They consisted of 20 negative (mean valence values in the 1-9 scale, 2.72; SD, 0.59 ) and 20 neutral images (mean valence values in the 1-9 scale, 5.07; $\mathrm{SD}, 0.63$ ). Emotional valence had been checked previously on 12 unrelated Belgian subjects. Each of the valence categories contained a similar proportion of images of animals, objects and landscapes. No image included a human face. The luminance of the background images was checked and standardized.

The target items were female and male faces with a neutral expression. Faces with distinctive features (beard, glasses, etc.) were discarded. The size of the faces was standardized and they were presented on a white background. Two lists of 40 faces with equal gender ratios were constructed. For each subject, one of these lists was randomly chosen for encoding. One-half of the faces of the second list were further used as distracters for the retrieval session. The pairings of the faces with the background images were fully randomized across subjects.

Between the trials, a fixation cross $\left(3.75^{\circ} \times 3.75^{\circ}\right)$ was displayed on a light background. The latter ensured a relative pupillo-constriction, allowing a better detection of pupillary dilatation related to stimulus presentation.

Data acquisition. Data were acquired with a $3 \mathrm{~T}$ head-only magnetic resonance (MR) scanner (Allegra; Siemens, Erlangen, Germany) using a gradient echo-planar imaging (EPI) sequence [32 transverse slices with $30 \%$ gap; voxel size, $3.4 \times 3.4 \times 3.4$; repetition time (TR), $2130 \mathrm{~ms}$; echo time (TE), $40 \mathrm{~ms}$; flip angle, $90^{\circ} ; 220 \mathrm{~mm}$ field of view (FOV)]. Shimming was performed before each EPI session by using a homemade procedure, ensuring better field homogeneity and higher signal-to-noise ratio in the amygdala and brainstem areas. Between 180 and 230 functional volumes were acquired during encoding and between 205 and 225 functional volumes were acquired during retrieval. In all sessions, the first three volumes were discarded to account for magnetic saturation effects. A structural MR scan was acquired at the end of the experimental session (T1-weighted three-dimensional magnetization-prepared rapid acquisition gradient echo sequence; TR, $1960 \mathrm{~ms}$; TE, $4.43 \mathrm{~ms}$; inversion time, $1100 \mathrm{~ms}$; FOV , $230 \times 173 \mathrm{~mm}^{2}$; matrix size, $256 \times 192 \times 176$; voxel size, $0.9 \times 0.9 \times 0.9 \mathrm{~mm})$. Stimuli were displayed on a screen positioned at the rear of the scanner, which the subject could comfortably see through a mirror mounted on the standard head coil.

Functional MRI data analysis. Functional MRI data from the retrieval session were analyzed using SPM2 (http://www.fil.ion.ucl.ac.uk) implemented in MATLAB (Mathworks, Sherbom, MA). Functional scans were realigned using iterative rigid body transformations that minimize the residual sum of square between the first and subsequent images. They were normalized to the Montreal Neurological Institute (MNI) EPI template (two-dimensional spline; voxel size, $2 \times 2 \times 2$ ) and spatially smoothed with a Gaussian kernel with full width at half-maximum (FWHM) of $8 \mathrm{~mm}$.

Data were processed using two-step analysis, taking into account the intraindividual and interindividual variance, respectively. For each subject, brain responses were modeled at each voxel, using a general linear model. During retrieval, four trial types were modeled, based on the emotional valence of the context at encoding and the subsequent memory effect: (1) correctly recognized faces encoded in emotional context ("neg rec"), (2) correctly recognized faces encoded in neutral context ("neu rec"), (3) faces encoded in emotional context subsequently forgotten ("neg forg"), (4) faces encoded in neutral context subsequently forgotten ("neu forg"). Two additional trial types consisted of the new faces, correctly identified as such or not. For each trial type, each single event was modeled as a $\delta$ function representing its onset. The ensuing vector was convolved with the canonical hemodynamic response function, and used as a regressor in the individual design matrix. Moreover, linear modulations of the brain response to each trial type by the pupillary size, recorded during encoding and during retrieval, was included in the design matrix. The pupillary size was estimated from the eye tracking recording, as described below. Movement parameters estimated during realignment (translations in $x, y$, and $z$ directions and rotations around $x$, $y$, and $z$ axes) and a constant vector were also included in the matrix as a variable of no interest. Linear contrasts estimated the main effect of memory (recognized vs forgotten), and emotion (negative vs neutral). However, the contrast of interest was essentially the memory by emotion interaction [i.e., (Neg Rec-Neu Rec) - (Neg For - Neu For)]. The same contrasts were assessed for responses modulated by the pupillary size (as recorded at encoding for the corresponding face). High-pass filter was implemented using a cutoff period of $128 \mathrm{~s}$ to remove the low-frequency drifts from the time series. Serial autocorrelations were estimated with a restricted maximum likelihood algorithm using an autoregressive model of order 1 ( + white noise). The resulting set of voxel values constituted a map of $t$ statistics [SPM(T)]. Because no inference was performed at the individual level, this map was thresholded at $p=0.90$ (uncorrected). The individual summary statistical images were spatially smoothed with a Gaussian kernel of $6 \mathrm{~mm}$ FWHM and used in a second-level analysis, corresponding to a random-effects analysis. The second-level analysis consisted of a one-sample $t$ test. The resulting set of voxel values was thresholded at $p=0.001$ (uncorrected). Statistical inferences were corrected for multiple comparisons using Gaussian random field theory at the voxel level in small spherical volumes (radius, $10 \mathrm{~mm}$ ) around a 
priori locations of structures of interest, taken from the literature (see Table 2).

This analysis was separately conducted in two groups of subjects: those exhibiting a better retrieval of faces encoded in emotional context (16 learners) and participants showing no emotional effect at retrieval (14 nonlearners) (see Results). We masked exclusively the maps obtained in learners by the results of the nonlearners (threshold $p<0.05$, uncorrected). This maneuver ensured that the identified responses were specific to learners.

Psychophysiological interaction (PPI) analyses were computed to test the hypothesis that functional connectivity of the seed area (amygdala) with the rest of the brain would differ between the remembered stimuli encoded in neutral or in emotional context. For each individual, the coordinates of the seed area corresponded to the local maxima detected within $4 \mathrm{~mm}$ of the peak voxel of the random-effects analysis.

A new linear model for PPI analyses was prepared at the individual level, using three regressors. One regressor represented the emotional status of hits (emotional vs neutral) modulated by the pupillary size. The second regressor was the activity in the reference area. The third regressor represented the interaction of interest between the first (psychological) and the second (physiological) regressors. To build this regressor, the underlying neuronal activity was first estimated by a parametric empirical Bayes formulation, combined with the psychological factor and subsequently convolved with the hemodynamic response function (Gitelman et al., 2003). The model of connectivity also included movement parameters. A significant psychophysiological interaction indicated a change in the regression coefficients between any reported brain area and the reference region, related to the retrieval of emotional (rather than neutral) correctly remembered stimuli. Next, individual summary statistic images obtained at the first-level (fixed-effects) analysis were spatially smoothed ( $6 \mathrm{~mm}$ FWHM Gaussian kernel) and entered in a second-level (random-effects) analysis using one-sample $t$ tests. Inferences were conducted as for the main effect analysis.

In the random-effect analyses, we computed posterior probability maps (PPMs) enabling conditional or Bayesian inferences about regionally specific effects (Friston and Penny, 2003). PPMs were computed for responses to the main effect of emotion and the memory by emotion interaction, both modulated by the pupillary size. We wanted to verify that responses presented a higher probability of response in the interaction than in the main effect of emotion, which would speak against any major confounding effects of heart rate (see Results).

Pupillary size data. During both fMRI sessions, eye movements and pupillary size were measured continuously using an infrared eye tracking system (LRO5000; ASL, Bedford, MA) (sampling rate, $60 \mathrm{~Hz}$ ). Trials contaminated by blinks were discarded. Mean pupillary size was estimated for the last second of the display of the face during encoding or retrieval sessions (Fig. 2). During this interval, the pupillary size was stable enough to assess the autonomic arousal during face processing. At encoding, the autonomic arousal was expected to reflect primarily the valence of the background image.

To validate the autonomic effect of the pupillary size, 12 different subjects were asked to score the valence [from 1 (neutral) to 4 (unpleasant)] and the intensity [from 1 (calm) to 4 (excitement)] of emotion during the presentation of the background images alone, while their pupillary size was being measured. In this control population, the pupillary size was significantly and linearly related to the subjective rates during the presentation of the background images (valence: mean of the correlation coefficients for 12 subjects, $r=0.35, p=0.00001$; intensity: mean of the correlation coefficients for 12 subjects, $r=0.33, p=$ 0.00008 ).

\section{Results}

\section{Behavioral results}

Because we wanted to characterize the responses to successful retrieval of emotional memories, data were collected until a sufficient number of subjects showed a better retrieval of faces encoded in emotional context (see Discussion). Sixteen (of 30) subjects were eventually included in the analysis. Except for the
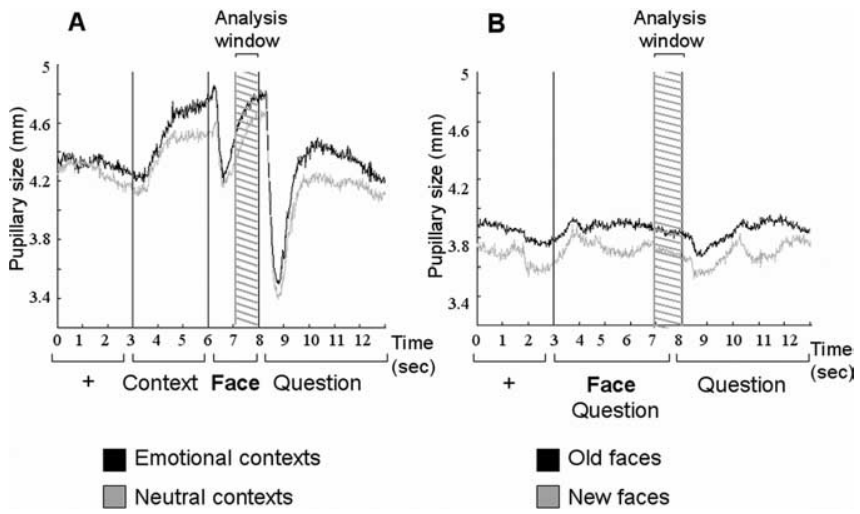

Figure 2. Mean pupillary changes for different trial types in a typical subject. $A$, Encoding session. The pupillary size is stable during baseline (fixation cross). During context presentation, the arousal induced by the context images influences the pupillary size. Face presentation induces a decrease in pupillary size because of both accommodation and light reflex (related to the white background of face images). Pupil dilatation then stabilizes, allowing a reliable measure. The average pupillary size recorded in the analysis window was introduced in the fMRI data analysis. $\boldsymbol{B}$, Retrieval session. The pupillary measure is stable during the presentation of the face and the questions.

Table 1. Behavioral data (SD)

\begin{tabular}{lcc}
\hline & Emotional & Neutral \\
\hline Hit accuracy (\%) & $61.9(19.6)$ & $49.1(17.2)$ \\
Source accuracy (\%) & $54.6(11.9)$ & $54.0(15.9)$ \\
Pupillary size at encoding $(\mathrm{mm})$ & $3.64(0.75)$ & $3.58(0.75)$ \\
Pupillary size at retrieval $(\mathrm{mm})$ & $3.40(0.60)$ & $3.41(0.62)$ \\
\hline
\end{tabular}

memory scores, these subjects did not differ from the excluded ones by any relevant parameter (age, sex, depression, or alexithymia scores; values of $p>0.05$ ). Expectedly, mean hit rates for stimuli encoded in an emotional context were higher than for stimuli encoded in a neutral context (paired $t$ test; $t_{(15)}=-7.02$; $p<0.001$ ) (Table 1). False alarm rate was $10.9 \pm 9.5 \%$. Correct rejection rate for old items was $85.9 \pm 11.1 \%$.

Source memory rate represents the proportion of context correctly remembered as emotional or neutral on correspondent hits items. There was no significant difference between source accuracy for emotional and neutral contexts $\left(t_{(15)}=\right.$ $-0.15 ; p=0.8$ ).

Pupillary size, taken as an index of emotional arousal, was monitored during both sessions. As expected, during encoding, pupillary size for recognized faces was significantly larger when they were encoded in a negative than in a neutral context $\left(t_{(15)}=\right.$ 2.62; $p=0.019$ ) (Table 1). In contrast, during the retrieval session, during which only neutral faces were presented, there was no significant difference in pupillary size for correctly retrieved faces encoded in emotional or in neutral context $\left(t_{(15)}=-0.27 ; p=0.8\right)$. However, pupillary size was significantly larger for hits (both emotional and neutral items; $3.41 \pm 0.61)$ than for correct rejection $\left(3.36 \pm 0.59 ; t_{(15)}=\right.$ $-0.53 ; p=0.01$ ) during retrieval.

\section{fMRI results}

Because our main objective was to characterize the brain responses to successful retrieval of emotional memories, we exclusively present the retrieval data in the following sections.

\section{Memory effect}

Analysis of old items that were correctly remembered (hits) versus old items that were forgotten (misses) revealed significantly 
Table 2. Functional MRI results

\begin{tabular}{|c|c|c|c|c|c|}
\hline Brain regions & Side & MNI coordinates (mm) & Zscore & $p_{\text {svc }}$ & References (coordinates found in) \\
\hline \multicolumn{6}{|l|}{ Effect of memory } \\
\hline Dorsolateral prefrontal cortex & L & $-48,26,24$ & 6.13 & $<0.001^{*}$ & \\
\hline Dorsolateral prefrontal cortex & $\mathrm{R}$ & $52,34,20$ & 3.24 & 0.045 & Kensinger and Corkin, 2004 \\
\hline Frontopolar cortex & L & $-26,62,2$ & 5.16 & $0.002^{*}$ & \\
\hline Middle frontal gyrus & $\mathrm{R}$ & $32,38,30$ & 3.62 & 0.016 & Smith et al., 2004 \\
\hline Middle frontal gyrus & $\mathrm{L}$ & $-40,46,-2$ & 4.71 & $<0.001$ & Maratos et al., 2001 \\
\hline Inferior parietal cortex & $\mathrm{L}$ & $-40,-54,-48$ & 4.12 & 0.004 & Smith et al., 2004 \\
\hline Lingual gyrus & $\mathrm{L}$ & $-24,-82,-12$ & 5.93 & 0.003 & Smith et al., 2004 \\
\hline Posterior cingulate & $\mathrm{L}$ & $-6,-36,30$ & 4.29 & 0.002 & Smith et al., 2004 \\
\hline Anterior cingulate & $\mathrm{L}$ & $-10,34,20$ & 3.24 & 0.045 & Maratos et al., 2001 \\
\hline Insula & $\mathrm{R}$ & $30,26,-4$ & 4.33 & 0.002 & Phan et al., 2004 \\
\hline \multicolumn{6}{|l|}{ Effect of emotion } \\
\hline Orbitofrontal cortex & $\mathrm{R}$ & $8,32,-18$ & 3.32 & 0.034 & Morris et al., 2004 \\
\hline Thalamus & R & $8,-12,-2$ & 3.21 & 0.045 & Fitzgerald et al., 2004 \\
\hline \multicolumn{6}{|c|}{ Interaction between emotion and memory } \\
\hline Amygdala & $\mathrm{R}$ & $8,-6,-24$ & 3.71 & 0.014 & Hamann et al., 1999 \\
\hline Parahippocampus gyrus & $\mathrm{L}$ & $-16,-24,-18$ & 3.37 & 0.035 & Smith et al., 2004 \\
\hline Middle temporal sulcus & $\mathrm{R}$ & $46,-2,-26$ & 3.49 & 0.026 & Smith et al., 2004 \\
\hline Inferior frontal cortex & $\mathrm{R}$ & $62,24,8$ & 3.35 & 0.037 & Smith et al., 2004 \\
\hline \multicolumn{6}{|c|}{ Interaction between emotion and memory, modulated by the pupillary size } \\
\hline Locus ceruleus & $\mathrm{R}$ & $2,-32,-20$ & 4.27 & 0.004 & Liddell et al., 2005 \\
\hline Locus ceruleus & $\mathrm{R}$ & $6,-36,-24$ & 3.74 & 0.021 & Liddell et al., 2005 \\
\hline \multicolumn{6}{|l|}{ Psychophysiological interaction } \\
\hline Locus ceruleus & $\mathrm{R}$ & $6,-34,-28$ & 3.21 & 0.048 & Coordinate of the interaction \\
\hline
\end{tabular}

R, Right; L, left.

*Regions significant after correction for multiple comparisons over the entire brain volume.

A

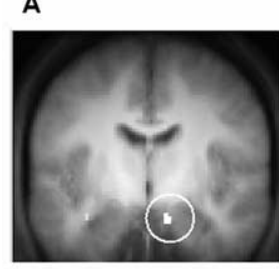

C
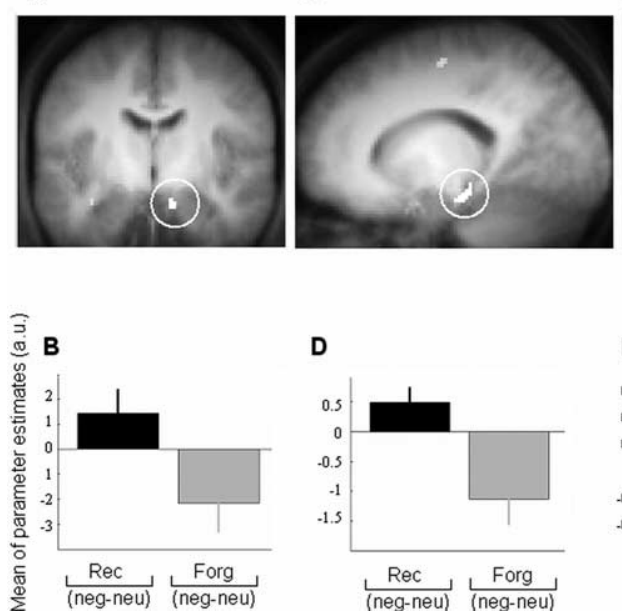

D

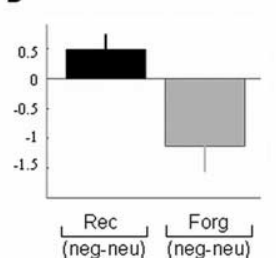

$\mathbf{E}$

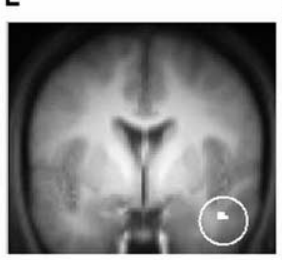

$\mathbf{F}$

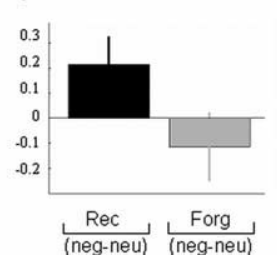

Figure 3. fMRI data. Significant responses during successful recognition of faces encoded in emotional context are shown. Top panels, Functional results are displayed on the mean structural MR image, normalized to the same stereotactic space (display at $p<0.001$, uncorrected). Bottom panels, Corresponding parameter estimates (arbitrary units $\pm \mathrm{SD}$ ). neu, Neutral; neg, emotional; Rec, recognized faces; Forg, forgotten faces. $\boldsymbol{A}, \boldsymbol{B}$, Right amygdala. C, D, Parahippocampus gyrus (display at $p=0.005$, uncorrected). $\boldsymbol{E}, \boldsymbol{F}$, Middle temporal sulcus. $\boldsymbol{G}, \boldsymbol{H}$, Inferior frontal cortex.

larger responses in a large frontal region including the dorsolateral prefrontal and the frontopolar cortex, a large occipitoparietal region including the precuneus and the superior parietal cortex, the lingual gyrus, the anterior and posterior cingulate cortex and the insula (Table 2).

G

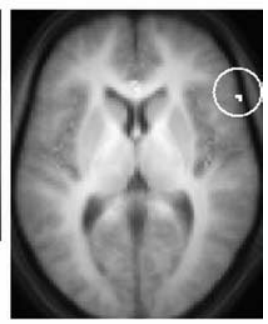

H

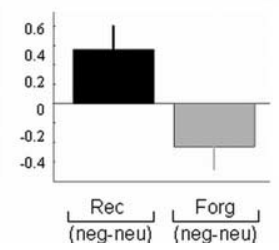

\section{Effect of emotion}

The main effect of emotion characterizes the responses to faces encoded in negative versus neutral context, independently of any memory effect. Faces elicited larger responses when they had been encoded in emotional rather than neutral context in the frontopolar cortex, the superior temporal cortex, the posterior cingulate cortex, the orbitofrontal cortex, and the thalamus (Table 2).

\section{Interaction between memory and emotion}

More importantly, we examined whether there was any difference in response for remembered versus forgotten items, depending on whether they had been encoded in an emotional or neutral context. A significant emotion by memory interaction was observed in the amygdala, the parahippocampal cortex, the middle temporal sulcus, and the inferior frontal cortex (Fig. 3, top panels; Table 2). As shown by their parameter estimates, these areas respond more to faces successfully recognized than forgotten, and more so when they are encoded in emotional than neutral context (Fig. 3, bottom panels). 

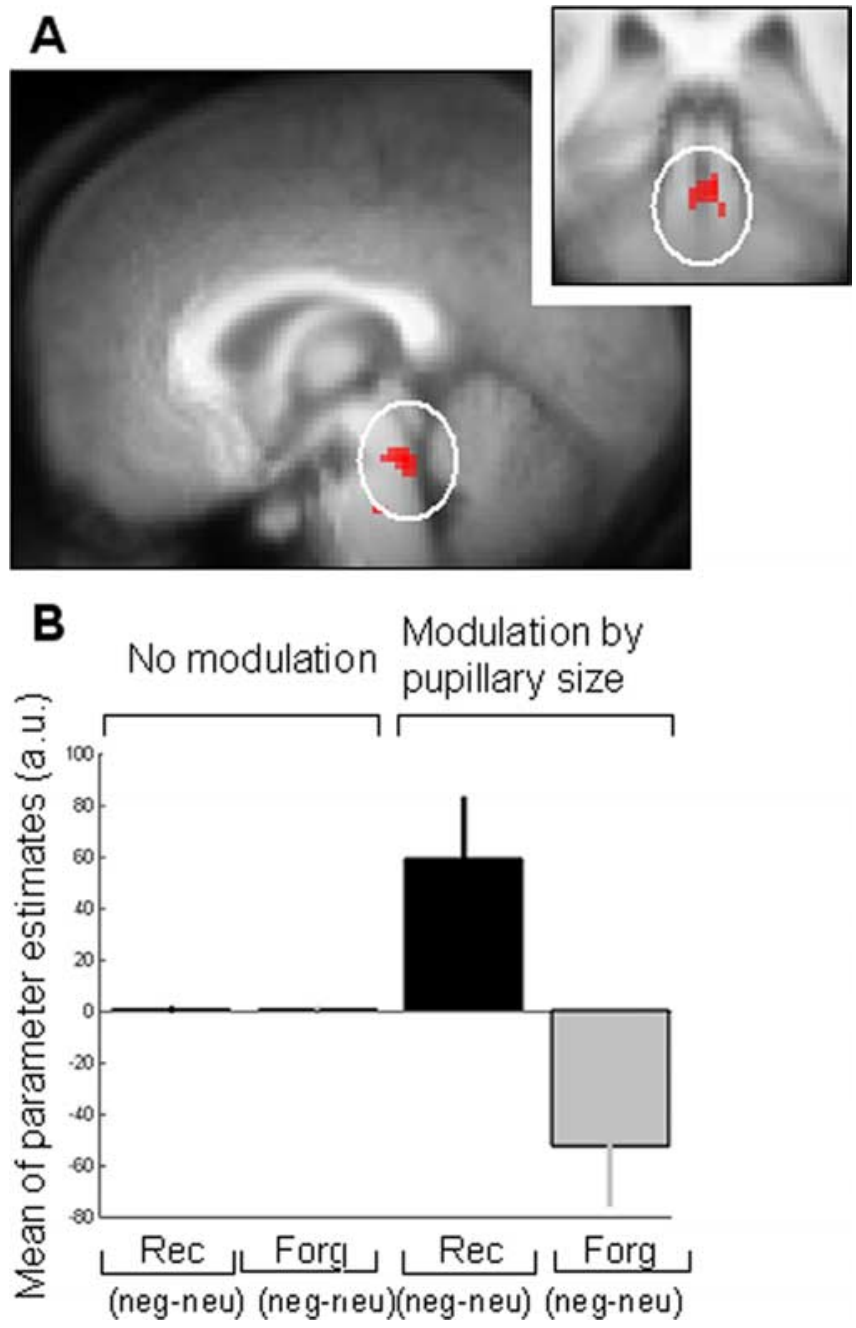

Figure 4. fMRI data. Significant responses during successful recognition of faces encoded in emotional context, modulated by the pupillary size recorded at encoding. $\boldsymbol{A}$, Locus ceruleus, displayed on the mean structural MR image, normalized to the same stereotactic space. $\boldsymbol{B}$ Parameter estimates of the responses in the $\mathrm{LC}$, modulated by the pupillary size observed at encoding (arbitrary units \pm SD).

\section{Interaction between memory and emotion modulated by the pupillary size}

To test whether the brain responses induced during retrieval were related to the autonomic arousal level at encoding, we introduced the pupillary size measured at encoding as a modulator of brain responses recorded during the retrieval session. A significant emotion by memory interaction was observed on brain responses linearly modulated by the pupillary size in an area of the dorsal tegmentum of the ponto-mesencephalic region, consistent with the location reported for the locus ceruleus (LC) (Liddell et al., 2005) (Fig. 4A). From now on, this area will be referred to as the LC. As shown by the parameter estimates, this area responds to the recognized faces encoded in negative context versus neutral context but only in proportion to the arousal observed at encoding (Fig. $4 B$ ). To verify that this region is specific to subjects showing an enhancement in behavioral performance (learners), we masked exclusively the interaction modulated by the pupillary size with the same contrast obtain in nonlearners. We observed an significant activation in the $\mathrm{LC}\left(x, y, z=2,-32,-20 ; p_{\mathrm{svc}}=\right.$ $0.004)$. These findings indicated that the activation of this region is specific to the subjects showing an emotional bias at retrieval.
Finally, because emotions are multidimensional processes that associate autonomic, hormonal, and neuronal responses, we cannot rule out that the brainstem activation was not related to a change in heart rate, for instance in the parabrachial nucleus. Indeed, we did not record heart rate and did not explicitly model it. Nevertheless, the confounding effect of heart rate on our results is unlikely. The interaction [ ( rec neg - for neg) - (rec neu - for neu)] protect us against a main effect of emotion. Moreover, if the identified brainstem response was related to heart rate, it should precisely appear in the main effect of emotion (neg-neu). Bayesian inference shows that the significant brainstem response had a lower posterior probability of being activated in the latter contrast $(8 \%)$ than in the interaction of interest $(87 \%)$.

\section{Psychophysiological interaction}

A psychophysiological interaction using the amygdala as reference region identified the brain areas that are functionally connected with the amygdala more tightly when the correctly remembered faces are encoded in an emotional context than in a neutral context. Only one area was detected, again in an area compatible with the LC (Table 2). This area overlapped with the region detected in the emotion by memory interaction modulated by the pupillary size (Fig. 5).

\section{Discussion}

The neural correlates of successful retrieval of emotional memories were characterized in normal participants using eventrelated fMRI. The results confirm the involvement of the amygdala and mesiotemporal areas in the recognition of items encoded in an aversive rather than neutral context. The data reveal that an area of the brainstem compatible with the LC also responds significantly during the successful recognition of neutral events encoded in an emotional context. This response depends linearly on the autonomic arousal recorded during encoding, as assessed by the pupillary size. Moreover, psychophysiological interactions show that the amygdala and the LC are functionally more connected during the correct recognition of faces encoded in an emotional rather than a neutral context.

These results suggest that retrieval of emotional memories not only involve the interaction between the amygdala and the hippocampal formation but also the interplay between the amygdala and the brainstem. The response in the LC resulting from this interaction is related to the emotional arousal during encoding. Through its widespread projections to the forebrain, the LC might restore a state of central arousal similar to the encoding phase, thereby facilitating retrieval by way of an increase in forebrain responsiveness.

\section{Behavioral performances}

Given the pace and randomization of the stimulus presentation, and according to previous literature (Maratos et al., 2001; Smith et al., 2004), small behavioral effects were expected. For a given individual, the absence of an emotional memory bias may be attributed to various factors. Some authors have argued that highly emotional stimuli may divert attention from the neutral item to be learned, thereby compromising encoding (Smith et al., 2004). Others have observed that negative context images are less likely to enhance subsequent memory than positive contexts (Erk et al., 2003; Smith et al., 2004). Finally, a recognition task involves a more passive retrieval mode that would be less sensitive to emotional biases than a free-recall test (Erk et al., 2005).

In our case, we did not aim at accounting for emotional mem- 
ory retrieval in general but only at characterizing the neural correlates of this process when a significant emotional bias is observed behaviorally. Consequently, data were collected until a sufficient number of subjects showed a better retrieval for neutral faces encoded in an emotional compared with a neutral context.

With regard to memory for the context images, we did not observe a significant influence of emotion. These results differ from a previous report (Smith et al., 2005), in which negative contexts were more easily remembered than neutral ones. This discrepancy might reside in the use of different encoding strategies. In contrast to Smith et al. (2005), we did not explicitly ask our subjects to associate context and target items. The shallow encoding of the contexts probably explains our negative results.

\section{Pupillary measures}

At any given time, the diameter of the pupil changes with the amount of light reaching the retina, in relation to accommodation and in response to any modification of the balance between sympathetic and parasympathetic outflows (Giakoumaki et al., 2005).

Consequently, the mean luminance of the context images was carefully matched. Likewise, accommodation effort was similar for all images. Fluctuation of alertness, attention, or discomfort in the scanner was unlikely to affect the pupillary size because of the stimulus randomization. Importantly, the emotional valence and intensity of context images, as assessed by unrelated control subjects, was found to be significantly related to the pupillary size.

In consequence, during the encoding session, it can be argued that the difference in pupillary size between neutral and emotional contexts reflected their systematic difference in affective valence. Expectedly, we found a significantly larger pupillary size during the presentation of remembered faces encoded in an emotional context than in a neutral context during the encoding session.

During the retrieval, the pupillary size was higher for old faces than for new ones. It should be kept in mind that for items identified as "old" faces, subjects were further questioned about their source memory. Because a highly demanding task elicits pupillary dilatation (Siegle et al., 2003), this supplementary effortful task might explain the difference in pupillary size between the hits and the correct rejections. However, this effect did not influence our main contrast of interest, which focused on responses to old faces.

During the retrieval, no significant difference in pupillary diameter was observed between remembered faces encoded in emotional and neutral contexts. Because only neutral faces were presented during the testing session, we did not expect any significant difference. Because of the differential responses observed centrally, especially in the amygdala and the brainstem, larger amygdalar responses for remembered faces encoded in emotional context might have been accompanied by a corresponding change in pupillary diameter. Although we cannot definitively reject the hypothesis that these negative findings might be attributable to a lack of sensitivity of our measure, these results are in agreement with the hypothesis of a dissociation between the cerebral responses related to memory retrieval and autonomic responses mediated by the peripheral nervous system. In this hypothesis, successful retrieval of items previously encoded in emotional context would essentially induce a central neural response, without autonomic counterpart.

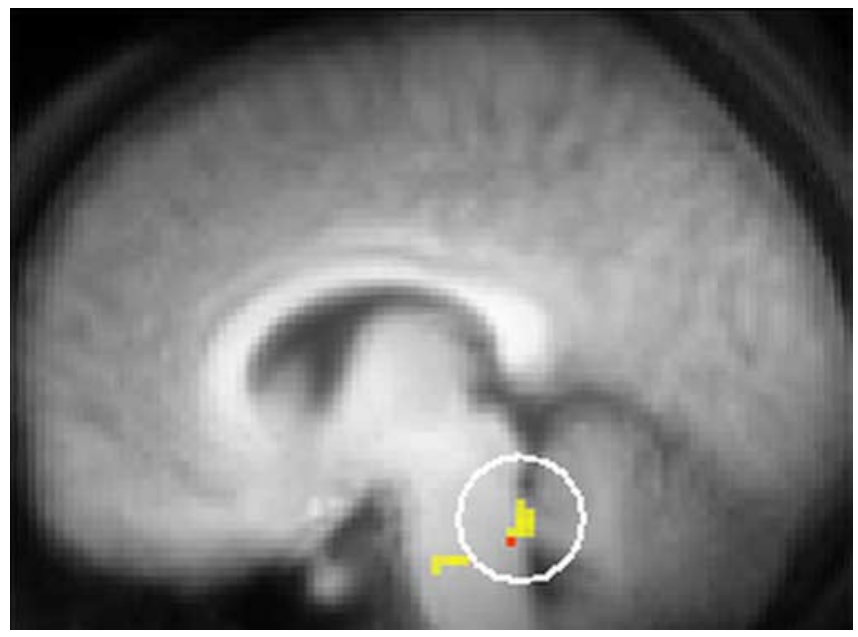

Figure 5. Psychophysiological interaction. PPI results (in red) overlap with the LC detected in the interaction contrast reported in Figure 4 (in yellow).

\section{Functional MRI data}

Our results confirm previous reports on the main effects of emotion and memory. Significant responses to recognized versus forgotten faces, regardless of their emotional contexts, were observed in several regions involved in memory retrieval: the dorsolateral prefrontal cortex (Henson et al., 2000), the frontopolar area (Henson et al., 2005) and parietal regions, precuneus and cingulate cortex (Taylor et al., 1998; Maguire et al., 2001; Lundstrom et al., 2005; Wagner et al., 2005). Likewise, a significant main effect of emotion (regardless of whether the face has been successfully retrieved or not) was detected in the orbitofrontal cortex, a region previously reported in similar experimental designs (Smith et al., 2004; Erk et al., 2005).

The interaction between emotion and memory supports the hypothesis of a modulation by the amygdala of the mesiotemporal areas. The amygdala is well known to be involved in emotional processes in general and in the retrieval of emotional memory in particular (Hamann, 2001).

A response pattern consistent with a significant emotion by memory interaction was observed in the parahippocampal gyrus. This area has been associated with subsequent memory effects at encoding (Cahill et al., 1996; Erk et al., 2003) and during retrieval (Smith et al., 2004), during which it is under direct amygdalar influence (Dolcos et al., 2004). In addition, successful retrieval of emotional memories recruits neocortical areas, notably the inferior frontal cortex. This cortical area interacts with the amygdala during the retrieval of intrinsically emotional stimuli (Kilpatrick and Cahill, 2003) and is involved in processing emotionally arousing visual stimuli and in emotional perceptual decision making (Pessoa and Padmala, 2005).

We hypothesized that the restoration of a central arousal similar to the one induced at encoding could participate in successful retrieval of emotional memories. Consequently, we correlated the brain responses during retrieval with the pupillary diameter measured during the encoding session. A significant (memory by emotion) interaction was observed in the LC. In other words, the responses in the LC were both linearly related to the arousal detected at encoding and larger for the recognized faces (compared with forgotten faces) if they had been encoded in negative context rather than neutral context. This LC response was specific to the subjects who showed an improvement of performance for faces encoded in emotional context. We further checked that 
the two main brain areas of interest identified during retrieval, the amygdala and the LC, were activated during the encoding. In keeping with our hypothesis, the analysis of the encoding session showed a significant main effect of emotional context on hits in the amygdala (coordinates, $26,-10,-14 \mathrm{~mm} ; Z=3.8 ; p_{\mathrm{svc}}=$ 0.009 ), and, at a more lenient threshold, in the LC when the responses are modulated by the pupillary size (coordinates, -6 , $-36,-26 \mathrm{~mm} ; Z=2.03 ; p_{\text {uncorrected }}=0.019$ ).

We next reasoned that the amygdala was in good position to restore the central arousal prevailing during encoding because it keeps track of emotional memories and it can influence the arousal through its projections to the hypothalamus and the brainstem. Using psychophysiological interactions, we showed that the amygdala and the LC were functionally connected during retrieval more tightly for items encoded in emotional than in neutral contexts. The interactions between the amygdala and the LC are probably bidirectional because these regions are mutually connected. There are afferent projections from the central nucleus of the amygdala to LC (Luppi et al., 1995). Conversely, the LC indirectly projects to the amygdala via the nucleus of the tractus solitarius (Berntson et al., 2003). These connections are proved to be functionally important (Bouret et al., 2003). Accordingly, the modulating influence of noradrenergic neurotransmission onto the amygdala takes part in emotional memory consolidation (Cahill and Alkire, 2003; Pare, 2003; McGaugh, 2004).

The coordinated action of the amygdala and the noradrenergic LC during retrieval might promote the activation of the (para)hippocampal region, thereby explaining the enhanced recall for emotional stimuli (Berntson et al., 2003; Bouret et al., 2003; Strange and Dolan, 2004).

\section{References}

Adolphs R, Cahill L, Schul R, Babinsky R (1997) Impaired declarative memory for emotional material following bilateral amygdala damage in humans. Learn Mem 4:291-300.

Beck AT, Ward CH, Mendelson M, Mock J, Erbaugh J (1961) An inventory for measuring depression. Arch Gen Psychiatry 4:561-571.

Bermond B, Vorst HC, Vingerhoets AJ, Gerritsen W (1999) The Amsterdam Alexithymia Scale: its psychometric values and correlations with other personality traits. Psychother Psychosom 68:241-251.

Berntson GG, Sarter M, Cacioppo JT (2003) Ascending visceral regulation of cortical affective information processing. Eur J Neurosci 18:2103-2109.

Bouret S, Duvel A, Onat S, Sara SJ (2003) Phasic activation of locus ceruleus neurons by the central nucleus of the amygdala. J Neurosci 23:3491-3497.

Cahill L, Alkire MT (2003) Epinephrine enhancement of human memory consolidation: interaction with arousal at encoding. Neurobiol Learn Mem 79:194-198.

Cahill L, Babinsky R, Markowitsch HJ, McGaugh JL (1995) The amygdala and emotional memory. Nature 377:295-296.

Cahill L, Haier RJ, Fallon J, Alkire MT, Tang C, Keator D, Wu J, McGaugh JL (1996) Amygdala activity at encoding correlated with long-term, free recall of emotional information. Proc Natl Acad Sci USA 93:8016-8021.

Critchley HD, Rotshtein P, Nagai Y, O’Doherty J, Mathias CJ, Dolan RJ (2005a) Activity in the human brain predicting differential heart rate responses to emotional facial expressions. NeuroImage 24:751-762.

Critchley HD, Tang J, Glaser D, Butterworth B, Dolan RJ (2005b) Anterior cingulate activity during error and autonomic response. NeuroImage 27:885-895.

Davis M (1992) The role of amygdala in conditioned fear. In: The amygdala: neurobiological aspects of emotion, memory, and mental dysfunction (Aggleton JP, ed), pp 255-305. New York: Wiley.

Davis M, Whalen PJ (2001) The amygdala: vigilance and emotion. Mol Psychiatry 6:13-34.

Dolan RJ (2002) Emotion, cognition, and behavior. Science 298:1191-1194.

Dolan RJ, Lane R, Chua P, Fletcher P (2000) Dissociable temporal lobe activations during emotional episodic memory retrieval. NeuroImage 11:203-209.

Dolcos F, LaBar KS, Cabeza R (2004) Interaction between the amygdala and the medial temporal lobe memory system predicts better memory for emotional events. Neuron 42:855-863.

Erk S, Kiefer M, Grothe J, Wunderlich AP, Spitzer M, Walter H (2003) Emotional context modulates subsequent memory effect. NeuroImage 18:439-447.

Erk S, Martin S, Walter H (2005) Emotional context during encoding of neutral items modulates brain activation not only during encoding but also during recognition. NeuroImage 26:829-838.

Fenker DB, Schott BH, Richardson-Klavehn A, Heinze HJ, Duzel E (2005) Recapitulating emotional context: activity of amygdala, hippocampus and fusiform cortex during recollection and familiarity. Eur J Neurosci 21:1993-1999.

Friston KJ, Penny W (2003) Posterior probability maps and SPMs. NeuroImage 19:1240-1249.

Giakoumaki SG, Hourdaki E, Grinakis V, Theou K, Bitsios P (2005) Effects of peripheral sympathetic blockade with dapiprazole on the fearinhibited light reflex. J Psychopharmacol 19:139-148.

Gitelman DR, Penny WD, Ashburner J, Friston KJ (2003) Modeling regional and psychophysiologic interactions in fMRI: the importance of hemodynamic deconvolution. NeuroImage 19:200-207.

Hamann S (2001) Cognitive and neural mechanisms of emotional memory. Trends Cogn Sci 5:394-400.

Hamann SB, Ely TD, Grafton ST, Kilts CD (1999) Amygdala activity related to enhanced memory for pleasant and aversive stimuli. Nat Neurosci 2:289-293.

Henson RN, Rugg MD, Shallice T, Dolan RJ (2000) Confidence in recognition memory for words: dissociating right prefrontal roles in episodic retrieval. J Cogn Neurosci 12:913-923.

Henson RN, Hornberger M, Rugg MD (2005) Further dissociating the processes involved in recognition memory: an fMRI study. J Cogn Neurosci 17:1058-1073.

Kensinger EA, Corkin S (2004) Two routes to emotional memory: distinct neural processes for valence and arousal. Proc Natl Acad Sci USA 101:3310-3315.

Kilpatrick L, Cahill L (2003) Amygdala modulation of parahippocampal and frontal regions during emotionally influenced memory storage. NeuroImage 20:2091-2099.

Lang PJ, Bradley MM, Cuthbert BN (1999) International Affective Picutre System (IAPS): instruction manual and affective ratings. Gainesville, FL: The Center for Research in Psychophysiology, University of Florida.

LeDoux JE, Iwata J, Cicchetti P, Reis DJ (1988) Different projections of the central amygdaloid nucleus mediate autonomic and behavioral correlates of conditioned fear. J Neurosci 8:2517-2529.

Liddell BJ, Brown KJ, Kemp AH, Barton MJ, Das P, Peduto A, Gordon E, Williams LM (2005) A direct brainstem-amygdala-cortical "alarm" system for subliminal signals of fear. NeuroImage 24:235-243.

Lundstrom BN, Ingvar M, Petersson KM (2005) The role of precuneus and left inferior frontal cortex during source memory episodic retrieval. NeuroImage 27:824-834.

Luppi PH, Aston-Jones G, Akaoka H, Chouvet G, Jouvet M (1995) Afferent projections to the rat locus coeruleus demonstrated by retrograde and anterograde tracing with cholera-toxin B subunit and Phaseolus vulgaris leucoagglutinin. Neuroscience 65:119-160.

Maguire EA, Frith CD, Cipolotti L (2001) Distinct neural systems for the encoding and recognition of topography and faces. NeuroImage 13:743-750.

Maratos EJ, Dolan RJ, Morris JS, Henson RN, Rugg MD (2001) Neural activity associated with episodic memory for emotional context. Neuropsychologia 39:910-920.

McGaugh JL (2004) Amygdala: role in modulation of memory storage. In: The amygdala. A functional analysis (Aggleton JP, ed), pp 391-423. New York: Oxford UP.

Morris JS, Friston KJ, Buchel C, Frith CD, Young AW, Calder AJ, Dolan RJ (1998) A neuromodulatory role for the human amygdala in processing emotional facial expressions. Brain 121:47-57.

Pare D (2003) Role of the basolateral amygdala in memory consolidation. Prog Neurobiol 70:409-420.

Pessoa L, Padmala S (2005) Quantitative prediction of perceptual decisions 
during near-threshold fear detection. Proc Natl Acad Sci USA 102:5612-5617.

Phelps EA, O’Connor KJ, Gatenby JC, Gore JC, Grillon C, Davis M (2001) Activation of the left amygdala to a cognitive representation of fear. Nat Neurosci 4:437-441.

Pitkanen (2004) Connectivity of the rat amygdaloid complex. In: The amygdala. A functional analysis (Aggleton JP, ed), pp 31-115. New York: Oxford UP.

Richardson MP, Strange BA, Dolan RJ (2004) Encoding of emotional memories depends on amygdala and hippocampus and their interactions. Nat Neurosci 7:278-285.

Siegle GJ, Steinhauer SR, Stenger VA, Konecky R, Carter CS (2003) Use of concurrent pupil dilation assessment to inform interpretation and analysis of fMRI data. NeuroImage 20:114-124.

Smith AP, Henson RN, Dolan RJ, Rugg MD (2004) fMRI correlates of the episodic retrieval of emotional contexts. NeuroImage 22:868-878.
Smith AP, Henson RN, Rugg MD, Dolan RJ (2005) Modulation of retrieval processing reflects accuracy of emotional source memory. Learn Mem 12:472-479.

Strange BA, Dolan RJ (2004) Beta-adrenergic modulation of emotional memory-evoked human amygdala and hippocampal responses. Proc Natl Acad Sci USA 101:11454-11458.

Taylor SF, Liberzon I, Fig LM, Decker LR, Minoshima S, Koeppe RA (1998) The effect of emotional content on visual recognition memory: a PET activation study. NeuroImage 8:188-197.

Wagner AD, Shannon BJ, Kahn I, Buckner RL (2005) Parietal lobe contributions to episodic memory retrieval. Trends Cogn Sci 9:445-453.

Williams LM, Phillips ML, Brammer MJ, Skerrett D, Lagopoulos J, Rennie C, Bahramali H, Olivieri G, David AS, Peduto A, Gordon E (2001) Arousal dissociates amygdala and hippocampal fear responses: evidence from simultaneous fMRI and skin conductance recording. NeuroImage 14:1070-1079. 